Curt Lehmann: Ueber den Einfluss von Alkali und Säure etc.

Versuch 2: wie der vorige ausgefiuhrt, Athemfrequenz 60, Athemgrösse 520-560 ccm pro Min. 10 Tetani wie oben: Athemgrösse steigt auf $1000 \mathrm{ccm}$, geht in 9 Min. auf 640 zurück, steigt in Folge von Unruhe wieder an, bis sie nach weiteren 8 Min. auf 490 heruntergegangen ist. - Unterbindung der Nierengefässe, neue 10 Tetani, Ansteigen bis $940 \mathrm{ccm}$, Abfall ohne Störung in $8 \mathrm{Min}$. bis auf $490 \mathrm{ccm}$.

Die Resultate dieser beiden Versuche, die vollkommen mit einander übereinstimmen, zeigen, dass die Verhinderung einer möglicherweise stattfindenden Ausfuhr der bei Muskelarbeit sich bildenden dyspnoëerregenden Stoffe aus dem Blute ohne Einfluss auf die Dauer und die Stärke der Dyspnoë bleibt und sie berechtigen mithin in Verbindung mit dem ersten Versuche zu der Behauptung, dass diese Stoffe nicht durch den Harn ausgesehieden werden.

Man wird demnach anzunehmen haben, dass manes mit leicht oxydirbaren, während der Dyspnö̈ im Körper des Versuchsthieres selbst der Zerstörung an heimfallenden Stoffen zu thun hat.

Aus dem thierphysiologischen Laboratium der königl. landwirthschaftlichen Hochschule in Berlin.

\title{
Ueber den Einfluss von Alkali und Säure auf die Frregung des Athemcentrums.
}

Von

\section{Dr. Curt Lehmann,}

Professor an der landw. Hochschule.

Nicht mit Unrecht ist die Thätigkeit des Athemcentrums mit der Bewegung eines Pendels verglichen worden. Die in gesetzmässiger Aufeinanderfolge eintretenden Inspirations- und Exspirations-Bewegungen erscheinen als die entgegengesetzten Ausschläge des Pendels, welches von irgend einem Impuls getroffen, in dieser Weise die empfangene Kraft in Bewegung umsetzt. Es ist bekannt, dass im Organismus diese Athembewegungen von versehiedener Richtung aus den Anstoss erfahren können. Sensationen 
von den peripheren Nervenendigungen aus, jedes Schmerzgefühl beeinflusst sie, meist die Ausgiebigkeit erhöhend, desgleichen vermögen rein psychische Functionen in verschiedenem Grade die Respiration zu alteriren. Neben diesen nervősen Einflüssen kommt jedoch wesentlich noeb die Zusammensetzung und Beschaffenheit des Blutes, das die betreffenden Centralorgane umspïlt, in Betracht. Wenn hier von der Einwirkung toxischer Substanzen resp. solcher, welche nur unter besonderen anormalen Verbältnissen dem Blute beigemischt sind, abgesehen wird, so sind früher allgemein nur zwei Körper in Betracht gezogen worden, deren Menge im Blut regulatorisch für die Athembewegungen wären, nämlich der Sauerstoff und die Kohlensäure. In vorstehender Arbeit von $\mathrm{Zuntz}$ und Geppert wurde jedoch der Nachweis gefuilrt, dass diese Gase normal entschieden nicht allein in Betracht kommen können, sondern dass bei einem Vorgang, der am häufigsten im Leben die Respiration beschleunigt, der Muskelarbeit, niclit wie früher angenommen wurde Sauerstoffmangel resp. Kohlensäurereichthun den Anlass zu stärkerer Athmung geben, sondern andere nicht gasartige Stoffe, die mit dem venösen Blute die Muskeln verlassen, den Weg durch die Lungen unverändert zurücklegen können und somit, durch das arterielle Blut zu dem Centralnervensystem geführt, das Athemcentrum reizen. Genannte Verfasser äusserten sich nicht über die Natur dieser räthselhaften Stoffe, deren chemische Bestimmung auch gar nicht im Yersuchsplane lag. Wohl wurde die Möglichkeit erörtert, dass die vom Muskel producirte Substanz eine Säure wäre, durch Titration des Blutes tetanisirter Kaninchen auch die Abnahme der Alkalescenz nachgewiesen, allein mit Recht mochten die Versuchsansteller nicht ohne Weiteres die Behauptung aussprechen, dass es $\mathrm{n} u \mathrm{r}$ die Veränderungen der Blutalkalescenz bei der Arbeit wären, die das Athemcentrum erregten, um so weniger als sie fanden, dass im Gegensatz zum Kaninchen das arterielle Blut von Hunden bald nach starker Muskelarbeit keinen erheblich geringeren Kohlensäuregehalt zeigte, als das Ruheblut, und dies den Schluss nahe legt, dass, obgleich bei Hunden nach Muskelarbeit ebenfalls heftige Dyspnoë auftreten kann, doch nicht erheblich weniger alkalische Affinitäten im Blute vorhanden seien. Weiterhin ist die Möglichkeit vorhanden, dass der Muskel irgend einen Stoff bei der Arbeit producirt, der bereits in minimaler Menge heftig auf nervöse Substanzen, speciell das Athemcentrum einwirkt. 
Dann ist die weitere Möglichkeit nicht ausgeschlossen, dass auch bei dem hier in Frage kommenden Processe der Erregung des Athemcentrums zwar primäre Ursache und Erfolg leicht als einfache Thatsachen durch die Beobachtung zu constatiren sind, aber wie so oft bei der Complicirtheit der Vorg:inge im Organismus die nähere Erklärung der Erscheinung, die detaillirtere Darlegung des Causalnexus zwischen primärer Ursache und Enderfolg eine grosse Mannigfaltigkeit von Vorgängen zu berücksichtigen hat. Kurz, es dürfte vor der Hand die Annahme gerechtfertigt erscheinen, dass die normale Reizung des Athemcentrums bei der Muskelarbeit nicht allein erfolgt, etwa durch eine einzige chemische Substanz, die in das rom Muskel abstammende Blut tritt, sondern eine ganze Reihe von Veränderungen im Körper stattfinden, die alle mehr oder weniger auf den Endeffect: Erregung des Centrums wirken. Beispielsweise duifte doch oft ein directer Reiz auf nervöser Bahn vom Willen zugleich mit dem motorischen Impuls auch auf die Athmung erfolgen. Gewiss wird in gewissem Grade auch die Erwärmung des Blutes durch die Muskelarbeit das Athemcentrum in Mitleidenschaft zieben. Endlich bleibt noch die Frage offen: ist es ein oder sind es mehrere Stoffe, die im Arbeitsblut - wenn dieser kurze Ausdruck gestattet ist - das erregende Princip darstellen, resp. ist nicbt auch ein Stoff darunter, dessen erregende Wirkung durch mehrere seiner Qualitäten hervorgerufen wird. (Z. B. specifisch chemische Affinitäten zur Substanz von Ganglienzellen, der Reaction etc.)

Um über diese Fragen und damit über die Gesammtheit des die Athmung regulirenden Apparates Aufschluss zu erbalten, dürte weniger der analytische Weg - Untersuchung des die venösen Centralorgane umspülenden Blutes, deren Schwierigkeit auf der Hand liegt - als der synthetische geeignet erscheinen, indem man verschiedene hier als möglich in Betracht kommende Körper in den Kreislauf bringt und ibre Wirkung auf die Athemmechanik studirt. Es liegt nahe, hier trotz der oben citirten Befunde der Blutgasanalyse beim Hunde doch an Stoffe zu denken, welche die Reaction des Blutes ändern. Wir wissen, dass z. B. durch die Muskelarbeit die Reaction der Muskelfaser sauer wird; eine Reihe von Arbeiten beweisen, dass die hierbei auftretende Milchsäure durch das Blut ausgewaschen wird und somit das venöse Blut an SäureAffinitäten bereichert. Da im Blut bekanntlich die Oxydation 
Ueb. den Einfluss von Alkali u. Säure auf die Erregung des A themcentrums. 287

organischer Substanzen nur eine minimale ist, z. B. injicirte Kohlehydrate etc. fast ausschliesslich in den Geweben verbrannt werden, so muss die eventuell aufgenommene Milchsäure fast unverändert den kleinen Kreislauf passiren, durch die Carotiden dem Athemeentrum zuströmen und kann hier eine Erregung desselben hervorrufen.

So weit eine solche Erregung lediglich auf der Abschwächung der Alkalescenz des Blutes beruht, müsste sie durch ein sonst indifferentes Alkali paralysirt resp. wieder aufgehoben werden können.

Diesem Gedanken entsprang der Plan zu folgenden gleich mitzutheilenden Versuchen. Es wurde geprift, ob wirklich in bestimmter Weise die Athemthätigkeit der Thiere durch Injectionen saurer und alkalischer Stoffe in die Blutbahn gesteigert resp. herabgesetzt werden könnte. Es wurden ausschliesslich Kaninchen bei den Experimenten beniitzt. Die tracheotomirten Thiere athmeten durch Müller'sche Ventile und ibre durch eine Gasuhr ge. messene Exspirationsluft diente als Maassstab für die Erregung der Athemcentren.

Die ersten Versuche ergaben keine beweisenden Resultate, da die Anordnung derselben zu wenig andere Wirkungen als die der veränderten Blutreaction auf die $\Lambda$ themcentren ausschloss. Immerhin mögen sie mit wenigen Worten berübrt werden, da sie einige hier Jeicht in Betracht kommende Fehlerquellen offenbaren.

Versuch I. 8. 3. 83.

$2630 \mathrm{gr}$ schweres Kaninchen. Alkalische Flüssigkeit: 1/10 normale Lösung von phosphorsaurem Natron; saure Flüssigkeit: 1/10 normale Phosphorsäure. Die Injection erfolgte aus einer Bürette in die Vena facial. poster. nach dem Herzen zu.

Es zeigte in diesem Versuche die Phosphorsäure in Mengen von 0,14 Milligramm-Aequivalenten allẻrdings eine stärkere Vermehrung der Athemgrösse (um $55 \%$ ), als das alkalisch reagirende phosphorsaure Natron in Mengen von 0,40 Milligramm-Aequivalenten (um $35 \%$ ), es wirkte aber auch letzteres erregend.

Es lag: nun die Möglichkeit vor, dass bei dem vollständig wachen Thiere die mit den Injectionen verbundenen Sensationen psychomotorisch wirkten und die geringe Steigerung der Alkalescenz durch das phosphorsaure Natron ibercompensirten. Daher 
wurde in den nächsten beiden Versuchen (Versuch II und III vom 17. und 20. März 83) das stärker alkalisch reagirende koblensaure Natron und zwar in zehnmal stärkerer, nämlich normaler Lösung, d. h. ein Milligramm-Aequivalent per Cubikcentimeter, angewendet. Die Säuren, zuerst Phosphorsäure, dann Weinsäure, waren ebenfalls normal. Die Injectionen erfolgten mittelst calibrirter Spritze in das Herzende einer Carotis.

In diesen beiden Versuchen zeigen kohJensaures Natron und die beiden Säuren characteristisch verschiedene Wirkung auf die Athemcentren. Wührend das Alkali in der etwas concentrirten Form, in welcher es jedenfalls noch die Medulla erreichte, zuerst die Athemthätigkeit fast vollständig inhibirte, hierauf aber das Thier gleichsam als Reaction auf die momentane Erstickung längere Zeit andauernd sehr starke Respirationen ausführte (es zeigten sich die höchsten Werthe für die Athemgrösse), wirkten die Säuren lediglich die Athmung erregend, zugleich aber den ganzen Organismus ermattend, das Thier erschöpfend. Es wäre hierbei nur die Frage, ob dieser Effect durch nichts weiter als die veränderte Reaction des die Ganglienzellen umspulenden Blutes hervorgebracht wurde. Dies ist nicht anzunehmen: es ist vielmehr hier bereits eine Summe von Einzelursachen, hervorgerufen durch die Injectionen, vorhanden.

Die Ader, in welche die Injection vorgenommen wurde, zeigte bald eine grosse Brïchigkeit, eine veränderte Beschaffenheit des Gewebes. Hieraus folgt, dass zugleich die Gefässnerven stark afficirt worden sein mussten und deren Erregung nicht einflusslos auf die Athemerregung bleiben konnte. Es ist anzunehmen, dass das Alkali stärker afficirte, vielleicht weil es von geringerer Diffusionsfähigkeit local länger auf die Nervenendigung in grösserer Concentration wirkte, als die Säure, die sich schneller weiter verbreitet. Aus den weiter unten zu berichtenden Versuchen geht die local stärker reizende Wirkung des Alkalis deutlich hervor.

Dann ist trotz der vorsichtigen Injection kaum glaublich, dass sich die Injectionflüssigkeiten vollständig mit dem Blute mischten, ihre alkalischen und sauren Affinitäten vollständig mit den entgegengesetzten des Blutes ausglichen, ehe sie die nervösen Centralapparate erreichten. Daraus folgt aber, dass die betreffenden Ganglienzellen nicht nur dem Einfluss von Blut veränderter Reac- 
tion, sondern mehr oder weniger direct einem Alkali- resp. Säurereiz ausgesetzt wurden. Jede Alkalilösung und jede Säurelösung wirkt aber zerstörend auf die Gewebe, also reizend, und ebenso wie vorhin in Bezug auf die Gewebe der Ader, würden wir hier auf das Centralnervensystem eine andauerndere Wirkung des Alkalis als der Säure annehmen müssen; gerade so, wie eine mit einer Alkalilösung benetzte Wunde länger schmerzt, als wenn die Benetzung mit einer gleich starken Säure gesehehen ist. Aus einer der hier nicht destillirten Beobachtungen (9 in Versuch III) geht auch die anhaltendere Wirkung der Alkalilösung hervor.

Verfehlt wäre es jedenfalls, wie auch die späteren Versuche beweisen, die beobachteten Veränderungen der Respiration in der Weise erklären zu wollen, dass man sagte: plötzliche Veränderungen der Reaction des Blutes reizen stets das Athemcentrum, eine Vermehrung der Alkalescenz reizt aber stärker als eine Vermehrung der Acidität um die gleichen Aequivalente und so ergäbe sich von selbst, dass bei der Injection von Alkali zuerst ein Athemstillstand beobachtet wurde, indem anfänglich eine Ueberreizung und damit Chockwirkung stattfand.

Der nächste Versuch (Versuch IV) mag vollständig berichtet werden.

$$
\text { Versuch IV. 20. 4. } 83 \text {. }
$$

Grosses weibliches Kaninchen, 2140gr. Durchschneidung des Rückenmarks zwischen 2. und 4. Lendenwirbel. Canüle in die linke Art. cruralis central eingebunden. (Operationen mit wenig Blutverlust.) Beendigung der Operation $1 \mathrm{~h} 30$. Frwachen des Thieres aus der Narkose und Beginn der Respirationsbeobachtungen 4h 30 . Alkalische Flüssigkeit: Zweifach normale $\mathrm{CO}_{3} \mathrm{Na}_{2}$-Lösung; saure Flüssigkeit : Normal-Weinsäure. Ablesung alle 5 Sekunden an einer Gasuhr, die direct Cubikcentimeter anzeigte.

I. Athmung vor der Injection in Cubikcentimetern pro 5 Sekunden. $70,60,70,50,60,60-50,60,60,60,50,60-50,60,80,60,70,70-$ $80,70,60,60,60,60-60,50,60,50,70,60-60,50,60,70,50,70-$ $50,70,50,70,50,50-60,60,60$.

II. Injection von $1 \mathrm{ccm}$ Alkali ${ }^{1}$ ) in etwa 8 Sekunden. Ehe aber die Ader wieder abgeklemmt war und die Athembeobachtung fortgesetzt werden konnte, vergehen in Summa 55 Sekunden. Hierauf:

$180,60,45,115,50,90-70,70,60,80,60,60-90,40,80,50,60,80-$

1) Bei der Alkali-Injection fand ein krampfhaftes Strecken der hinteren Extremitäten statt, das nur allmählich abtönte; bei der ersten Injection stärker als bei der zweiten. 
$80,60,80,60,60,80-70,60,90,90,70,70-70,70,100,50,70,70-$ $60,80,80,50,90,60-70,70$.

III. Injection von $1 \mathrm{ccm}$ Alkali. Abermals vergehen 55 Sekunden, ehe die Beobachtung festgesetzt werden kann.

$20,130,20,90,50,90-100,80,90,110,80,80-80,80,100,60,90,60-$ $90,80,50,70,60,70-60,70,40,100,70,70-80,60,80,60,90,80-$ $70,100,90,80,90,90-90,80,80,80,80,80-60,80,80,50$.

Hierauf 20 Minuten Pause.

IV. Athmung ohne weitere Injection.

$60,60,60,60,60,60-60,60,60,60,60,70-70,50,60,60,70,60-$ $60,60,60,70,60,60-80,60,90,70,80$.

V. Injection von $1 \mathrm{ccm}$ Säure. Dauer 40 Sekunden. Hierauf Athmung. $110,120,100,150,100,100--140,100,100,100,100,100$ - 100,90, 110,110,110, 110 $120,100,110,100,100,100-90,110$.

VI. Ohne weitere Injection nach 135 Sekunden Pause.

$100,60,80,60,60,60-80,60,70,70,60,70-70,70,70,90,80,90-$ $70,80,90$.

VII. Injection von Säure. In Folge einer kleinen Verletzung der Ader gelangte aber nur wenig von dem Cubikeentimeter ins Blut. Dauer der Injection 40 Sekunden. Hierauf Athmung.

$60,90,100,140,70,90-60,100,70,70,90,90-70,90,70,70,90,80-$ $70,90,70,90,80,80-90,90,80,90,80,90$.

VIII. Nach 12 Minuten Pause und nochmaligem Einbinden der Canüle ohne Injection.

$70,90,80,70,70,70-90,70,90,70,80,70-90,80,80,80,110,80-$ $90,90,100,90,80,80-90,90,80,90,80,90$.

IX. Injection von Alkali. Die Ader ist aber so brüchig, dass der grösste Theil des Cubikcentimeters nicht ins Blut kommt. Dauer der Injection 45 Sekunden. Hierauf Athmung.

$80,100,120,100,110,100-90,100,80,80,90,80-80,70,80,90,70,80$ $80,70,80,70,80,70-90,70,80,70,80,90-100,80,80,100,100,100$ $70,100,80,100,90,90$.

In Versuch 4 waren durch die Trennung des Rückenmarkes alle nervösen Erregungen des Athemcentrums von der Injectionsstelle her ausgeschlossen, ferner dadurch, dass die injicirten Flüssigkeiten erst den Capillarkreislauf des einen Schenkels passiren mussten, die innige Untermengung derselben mit dem schliesslich dem Hirn zufliessenden Blute wohl gesichert.

Als die reine Wirkung des durch die Injection veränderten 
Ueb. den Einfluss von Alkali u. Säure auf die Erregung des A themcentrums, 291

Versuch IV.

\begin{tabular}{|c|c|c|c|c|c|c|c|c|c|}
\hline & 递 & & & hemgrö & össe in & 1 cc. & & Injeoticn & \\
\hline هं & 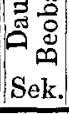 & 1. & & $\begin{array}{c}3 . \\
\text { Minute }\end{array}$ & 4. & 5. & $\begin{array}{c}\text { Mittel } \\
\text { pro } \\
\text { Min. }\end{array}$ & $\mid \begin{array}{c}\text { Milngramm- } \\
\text { Aequi- } \\
\text { valenten. }\end{array}$ & Bemerkungen. \\
\hline 1 & 230 & 710 & 780 & 710 & $(720)^{*} \mid$ & - & 730 & - & \\
\hline 2 & 220 & 940 & 820 & 880 & $(840)^{*}$ & - & 873 & $2.0 \mathrm{CO}_{3} \mathrm{Na}_{2}$ & $\begin{array}{l}\text { Beobachtung } 60^{\prime \prime} \text { nach } \\
\text { Beginn der Injection. }\end{array}$ \\
\hline 3 & 260 & 940 & 890 & 860 & 1010 & (810) & 916 & $2.0 \mathrm{CO}_{3} \mathrm{Na}_{2}$ & $\begin{array}{l}\text { Beobachtung } 55^{\prime \prime} \mathrm{p} . \\
\text { inj. Hierauf } 28 \mathrm{Min} . \\
\text { Pause. }\end{array}$ \\
\hline 4 & 145 & 730 & 740 & - & - & 一 & 765 & - & \\
\hline 5 & 130 & 1320 & 1260 & - & 一 & - & 1243 & 1.0 Säure & $\begin{array}{l}\text { Beobachtang } 40^{\prime \prime} \text { nach } \\
\text { Beginn der Injection. } \\
\text { Hierauf } 21 / 4 \text { Min.Pause. }\end{array}$ \\
\hline 6 & 105 & 830 & (943) & - & 一 & - & 880 & - & \\
\hline 7 & 150 & 1030 & 950 & $(1040)$ & - & - & 1000 & 0.3 Säure (?) & $\begin{array}{l}40^{\prime \prime} \text { p. inj. beobachtet. } \\
\text { Hierauf } 12 \text { Min. Pause. }\end{array}$ \\
\hline 8 & 150 & 920 & 1050 & $(1040)$ & - & - & 996 & - & \\
\hline 9 & 210 & 1130 & 920 & 1040 & $(1060)$ & - & 1034 & ? $0.3 \quad \mathrm{CO}_{3} \mathrm{Na}_{2}$ & $\begin{array}{l}\text { Injectionsdauer inclu- } \\
\text { sive Oeffnen und Ab- } \\
\text { klemmung der Ader } \\
45^{\star} \text {, hierauf beobachtet }\end{array}$ \\
\hline
\end{tabular}

Blutes zeigt sich demnach, dass sowohl durch $\mathrm{CO}_{2} \mathrm{Na}_{2}$, wie durch Weinsäure die Respirationsgrösse gesteigert wird, aber ganz entschieden durch die Säure erbeblich mehr. Vier Milligramm-Aequivalente kohlensaures Natron reizten das Athemcentrum weniger als ein Milligramm-Aequivalent Weinsäure. Hier war auch nichts von einer anfänglichen Chock-Wirkung des Alkalis zu bemerken, wohl aber zeigte sich (vergl. Beobachtung 3) im ganzen Innervationsgebiete des abgetrennten Rückenmarks eine starke, wohl reflectorisch hervorgebrachte Einwirkung auf die Muskeln, so lange nicht durch die wiederholten Injectionen die Erregbarkeit der Gewebe abgeschwächt war. Es ist bieraus zu erkennen, dass analoge Reizungen in den ersten Versuchen sicher an der Erregung der nervösen Centralorgane erheblich Antheil gehabt haben.

In welcher Weise ist nun die erregende Wirkung auch das kohlensauren Natrons zu erklären?

*) Die in dieser, wie in den folgenden Tabellen eingeklammerten Zahlen sind nicht dircet beobachtete, sondern aus der Athemgrösse in der letzten nicht vollständig beobachteten Minute auf die ganze Minute berechnet worden. 
Zuerst wird man daran zu denken haben, dass allein die Erhöhung des Salzgehaltes des Blates auf die Ganglienzellen reagiren musste. Der Gehalt von diffundiblen Salzen muss sich stets, so weit chemische Bindungen nicht in Betracht kommen, zwischen den Gewebselementen und dem Blute ins Gleichgewicht setzen. Jede Störung dieses Gleichgewichtes wird Diffusionsströme induciren, die ihrerseits nicht ohne Reizung der Zellen verlaufen können. Ingleichen wird ein Thier durch solche Processe, die momentan im ganzen Körper einsetzen, in seinem Allgemeingefühl alterirt werden, so dass sich zu der auf die Athemcentren direct erfolgenden Reizung noch ein psychomotorisches Element addirt. Jede Bewegung in letzterer Beziehung beschleunigt aber ebenfalls die Athmung. Wenn daher durch Injection alkalischer Salze und damit bewirkter Erböhung der Alkalescenz des Blutes die Erregung der Athemcentren herabgesetzt wird, wird diese Wirkung stets durch die eben erwähnten Momente paralysirt werden miissen.

Endlich ist zu erwähnen, dass das kohleusaure Natron gemischt mit dem Blute resp. den alkalisch reagirenden Geweben nicht ohne Weiteres und in jeder Richtung als eine alkalisch wirkende Materie anzusehen ist. Wie die Versuche von Pflüger, Zuntz, Gaule, Setschenow u. A. beweisen, verbält sich z. B. eine reine Lösung von diesem Salz ganz anders in Bezug auf die Bindung von $\mathrm{CO}_{2}$ als wenn sie mit BIut (Serum, Cruor oder beides) gemischt ist ${ }^{1}$ ). Die sauren Affinitäten der Bluteiweisse bringen sich zur Geltung und lockern die Bindung von $\mathrm{CO}_{2}$.

Gaule konnte direct nachweisen, dass die Spannung der Kohlensäure iaber Blat durch Zusatz von Natriumcarbonat bei Körpertemperatur vermehrt wurde. Es scheint bei dem letzten Versuche ausgeschlossen, dass bei Injection des Natronsalzes durch freie Kohlensäure die Athemcentra gereizt wurden, da das Blut mit dem Natronsalz erst den Lungenkreislauf passiren musste, ehe es zum Gehirn und Ruckenmark strömte, immerhin dürfte aber die Zerlegung durch die sauren Affinitäten des Hämoglobins etc. nicht so schnell erfolgen, dass nicht doch wenigstens die Ausspülung der im nervösen Gewebe erzeugten $\mathrm{CO}_{2}$ mangelhafter erfolgteJedenfalls, wie gleich mitzutheilende Versuche lehren, verhält sich das $\mathrm{CO}_{3} \mathrm{Na}_{2}$ anders, d. h. mehr erregend, als $\mathrm{NaOH}$.

1) Vergl. hierüber $N$. Zuntz: Physiologie der Blutgase etc. in $\mathrm{Her}^{-}$ manns Handbuch der Physiol. p. 64 u. s. w. 
In den nachstehenden drei Versuchen wurden die Thiere während der Athmung in leichter Aether-Narcose erhalten, um psychische Erregungen nach Möglichkeit zn vermeiden. Unterhalten wurde die Narcose dadurch, dass die inspirirte Luft, ehe sie in das erste Müller'sche Ventil trat, durch eine den Spritzflaschen ähnlich armirte Flasche streichen musste, deren Boden mit einer Aetherschicht überdeckt war. Das mit dem Inspirationsventil verbundene Glasrohr wurde so weit in die Flasche eingesenkt, dass die in die Lungen des Thieres gelangende Luft gerade genügend mit Aetherdämpfen gemiseht war. Die Narcose war nicht so tief, um Augenreflexe auszuschliessen, sondern liess das Thier nur für leichte Erschuitterungen, schwaches Kneifen etc. reactionslos erscheinen.

\section{Versuch V.}

$1050 \mathrm{gr}$ schweres Kaninchen. Durchschneidung des Rückenmarks am letzten Rückenwirbel. Fünf Stunden nach dieser Operation wurde in die linke Art. cruralis eine Canüle central eingebunden. Injection aus Bürette von Normal-NaOH und Normalweinsäure. Während des Versuches wurde das Thier in gleichmässiger leichter Aethernarkose erhalten. Ablesung alle 5 Sekunden. Angabe in Cubikcentimetern.

I. Athmung vor der Injection.

$35,35,30,45,35,40-35,40,30,40,30,40-40,35,30,35,40,30-$ $35,35,35,35,30,35-35,30,30,30,40,30-30,30,40,40,40,30-$ $35,35,30,35,25,40-40,30,30,30,40,30$.

II. Injection von $1,3 \mathrm{ccm}$ Alkali. $40,50,30,40,30,40-35,30,40,35,40,35-35$.

III. Injection von $1,5 \mathrm{cem}$ Alkali.

$40,40,50,40,40,40-40,30,20,25,25,40-40,20,30,30,30,30-$ $30,30,30,30,25,25-30,30,30,15,40,30-25$.

IV. Injection von $1,6 \mathrm{ccm}$ Alkali.

$30,35,30,30,35,30-30,30,20,30,25,30-20,25,15,30,20,25-$ $30,30,25,20,30,25-25,25,25,20,30,20-25,30,30,25,25,30-30$.

V. Injection von $2,1 \mathrm{ccm}$ Säure.

$30,40,50,30,40,50-50,70,55,55,90,70-70,60,80,70,50,80-$ $70,50,60,60,60,60-50,50,50,55,55$.

VI. Injection von $2,3 \mathrm{ccm}$ Alkali.

$40,40,45,45,50,35-55,40,50,40,40,35-30,40,30,40,40,40-$ $25,25,25,30,30,30-30$. 
VII. Injection von $1,8 \mathrm{ccm}$ Alkali. (Stossweises Athmen.)

$20,30,20,40,30,50-50,60,30,40,40,40-45,45,40,40,30,40-$ $50,30,30,30,35,35-20$.

VIII. Injection von $1,95 \mathrm{ccm}$ Säure.

$30,30,40,40,45,50-45,60,60,50,60,60-50,60,55,55,55,55-$ 50,60 .

IX. Injection von 2,05 ccm Säure. (Krampfhaftes, unterbrochenes Athmen, bald längere Athempausen und dann todt.)

$50,10,40,30,30,50-40,40,20,20,20.20-30,30,25,25,10,10 .-$

Versuch V.

\begin{tabular}{|c|c|c|c|c|c|c|c|c|}
\hline \multirow{2}{*}{ 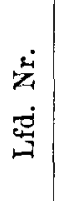 } & \multirow{2}{*}{$\begin{array}{c}\text { Dauer } \\
\text { der } \\
\text { Beob- } \\
\text { acht. } \\
\text { Sek. }\end{array}$} & \multicolumn{5}{|c|}{ Athemgrösse in ccm } & \multirow{2}{*}{$\begin{array}{c}\text { Injection } \\
\text { in } \\
\text { Milligramm- } \\
\text { Aequi- } \\
\text { valenten. }\end{array}$} & \multirow{2}{*}{ Bemerkungen. } \\
\hline & & 1. & $\begin{array}{l}2 . \\
\text { Min }\end{array}$ & $\begin{array}{r}3 . \\
\text { ate. }\end{array}$ & 4. & $\begin{array}{l}\text { Mittel } \\
\text { pro } \\
\text { Min. }\end{array}$ & & \\
\hline 1 & 240 & 435 & 415 & 405 & 400 & 413 & - & \\
\hline 2 & 65 & 445 & - & 一 & - & 443 & $1.3 \mathrm{NaOH}$ & \\
\hline 3 & 155 & 430 & 350 & - & - & 379 & $1.5 \mathrm{NaOH}$ & \\
\hline 4 & 185 & 355 & 295 & 310 & - & 321 & $1.6 \mathrm{NaOH}$ & \\
\hline 5 & 145 & 630 & 770 & - & - & 687 & 2.1 Woinsäure. & \\
\hline 6 & 125 & 515 & 385 & - & - & 446 & $2.3 \mathrm{NaOH}$ & \\
\hline 7 & 125 & 450 & 450 & - & - & 441 & $1.8 \mathrm{NaOH}$ & $\begin{array}{c}\text { Etwas stossweisses } \\
\text { Athmen. }\end{array}$ \\
\hline 8 & 100 & 570 & $(630)$ & - & - & 606 & 1.9 Woinsäure. & \\
\hline 9 & 90 & 370 & - & - & - & 333 & 2.1 Woinsïure. & $\begin{array}{l}\text { Krampfhaft unter- } \\
\text { brochenes Athmen. } \\
\text { - Einige Pausen. } \\
\text { Thier stirbt nach } \\
11 / 2 \text { Minuten. }\end{array}$ \\
\hline
\end{tabular}

Versuch VI. 21.6. 83.

Versuchsanordnung wie vorige. Kaninchen $1510 \mathrm{gr}$.

I. A thmung vor der Injection.

$60,50,60,50,45,45-50,45,45,50,45,45-50,50,50,35,40,45-$ $40,50,45,55,50,50-45,45,45,35,50,50-55,45,50,40$.

II. Injection von $0,55 \mathrm{ccm}$ Weinsäure.

$\left.65,75,60,90,100^{1}\right), 85-65,80,80,70,70,70-80,70,60,70,70,60$.

III. Injection von $0,99 \mathrm{ccm}$ Weinsäure. (Gleich darauf starke Bewegung und stürmische Athmung.)

$50,90,110,80,120,90-120,130,80,140,120,120-120,120,110,110,110,100$ -

1) Etwas Bewegung des Thieres. 
Ueb. den Einfluss von Alkali u. Säure auf die Erregung des Athemcentrums. 295

$85,95,90,90,90,100-80,90,90,90,80,70-95,95,80,80,80,100-$ $95,95,100,90,90,85-95,90,80,80,70,80-80,60,90,60,70,70$. -

Die beschleunigte Athmung hält noch einige Minuten an. Der Versuch hierauf Alkali zu injiciren misslang, da sich in Canüle und Ader sofort feste Gerinnsel bildeten.

\section{Versuch VII. 25. 6. 83.}

Operation und Behandlung des Kaninchens wie in den beiden vorigen Versuchen. $1360 \mathrm{gr}$ schwer.

\section{Athmung vor der Injection.}

$50,50,60,55,45,50-45,45,55,45,50,60-50,50,50,50,45,55-$ $50,50,60,55,55,50-60,60,45,50,55,60-60,60,65,65,55,55-$ $60,60,80,80^{*}, 70,50-60,60,50,60,60,60-60,65,65,60,60,55-$ $45,60,60,55,55,55-55,50,50,55,65,50-60,70,50,60,70,60-$ $70,60,70$.

* Zappeln des Thieres.

II. Injection von $1,95 \mathrm{cem}$ Alkali.

$65,65,70,70,60,60-70,60,60,60$.

III. Injection von $2 \mathrm{ccm}$ Alkali.

$50,60,50,60,70,90-50,60,60,55,55,65-55,60,50,40,50,60$.

IV. Injection von $1,9 \mathrm{ccm}$ Alkali.

$60,40,60,60,50,60-55,55,100,100^{*}, 70,80-70,80,70,70,70,65-$ $55,65,55,80,70,60-60,60$.

* Starke Unruhe des Thieres.

V. Injection von $2,15 \mathrm{ccm}$ Alkali.

$40,90,50,140^{*}, 90,90-80,70,70,60,80,70-80,60,80,60,60,70-$ $70,60,80,70,60,65-70,85,50,70,60,70-60,60,50,60,60,50-$ $55,60,55,50,50,55-50,55,50,60,50,50-50,50,50,50,50,50$.

Die Athmung verläuft in gleicher Weise noch $2 \frac{1}{2}$ Minuten fort.

* Starke Unruhe des Thieres.

VI. Injection von $1,6 \mathrm{ccm}$ Säure.

$60,90^{*}, 80,90,100,90-100,100,120,120,100,110-120,130,110,120,110,100$, $110,90,105,85,100,80-90,80,80,80,90,80-70,75,75,80$.

* Geringe Unruhe des Thieres.

VII. Injection von $1,8 \mathrm{ccm}$ Säure.

$90,80,80,90,100,120-140,130,150,120,140,120-120,120,110,110,110$, $60-150$.

VIII. Injection von $1,9 \mathrm{ccm}$ Alkali.

$105,85,100,90,100,90-70,100,90,80,85,95-80,80$.

IX. Injection von $1,35 \mathrm{ccm}$ Alkali.

$80,70,80,80,60,70-70,60,60,65,60,65-60,60,60,60,60,70-$ $50,70,50,60$. 
X. Injection von $2,1 \mathrm{ccm}$ Säure.

$70,60,80,70,100,100-110,130,110,160,140,140-150,120,130,130,130$.

XI. Injection von $1,75 \mathrm{ccm}$ Alkali. $120,120,130,120,100,110-120,120,120,130,110,140$.

XII. Injection von $0,8 \tilde{\mathrm{g}} \mathrm{cm}$ Alkali.

$130,120,110,130,130,110-100,110,110,110,100,100-105,105,90,100,100,100$ $100,105,95,110,120,110-105,105,110,100,100,100-100,100,100,100,100,80$. $100,100,95,85,90,95-100,100,95,95,95,110-110,90,110,90,100,100-$ 100,95 .

XIII. Injection von fast $2,5 \mathrm{ccm}$ Alkali.

$85,90,110,80,90,80-80,90,90,70,80,80-80,75,85,70,70,80 \ldots$ $70,70,90,70,80,70-70,70,80,70,80,70-60,70,60,60,70,60-$ $60,60,50,60,70,55-45,50,50,50,50,60$.

Versueb VII.

\begin{tabular}{|c|c|c|c|c|c|c|c|c|}
\hline $\begin{array}{l}\text { 㟒 } \\
\text { 岂 }\end{array}$ & $\begin{array}{l}\text { Dauer } \\
\text { der } \\
\text { Beob- } \\
\text { acht. } \\
\text { Sek. }\end{array}$ & 1. & $\begin{array}{l}2 . \\
\mathrm{Mi}\end{array}$ & $\begin{array}{r}3 . \\
\text { ute. }\end{array}$ & in ecn & iml & $\begin{array}{c}\text { Injection } \\
\text { in } \\
\text { Milligramm- } \\
\text { Acqui- } \\
\text { valenten. }\end{array}$ & Bemerkungen. \\
\hline 1 & 375 & 610 & 620 & 680 & $750^{*}$ & 680 & - & * Unruhe. \\
\hline 2 & 50 & $(768)$ & - & - & - & 768 & $1.95 \mathrm{NaOH}$ & \\
\hline 3 & 90 & 725 & $(630)$ & - & - & 693 & 2.00 & \\
\hline 4 & 130 & 990 & 810 & 一 & - & 794 & 1.9 & \\
\hline 5 & 420 & $930^{*}$ & 815 & 745 & 640 & 739 & $2.15 \quad "$ & $\begin{array}{l}\text { * Starke Unruhe } \\
\text { des Thieres. }\end{array}$ \\
\hline 6 & 170 & 1160 & 1260 & - & - & 1137 & 1.6 Weinsăuro & \\
\hline 7 & 95 & 1360 & (1337) & - & - & 1346 & 1.8 & \\
\hline 8 & 70 & 1090 & $(960)$ & - & - & 1071 & $1.9 \mathrm{NaOH}$ & \\
\hline 9 & 110 & 820 & $(720)$ & - & - & 774 & 1.35 & \\
\hline 10 & 85 & 1270 & $(1584)$ & - & - & 1362 & 2.1 Weinsầure & \\
\hline 11 & 60 & 1440 & - & - & - & 1440 & $1.75 \mathrm{NaOH}$ & \\
\hline 12 & 280 & 1350 & 1240 & 1200 & 1160 & 1231 & 0.85 & \\
\hline 13 & 240 & 1025 & 910 & 820 & 660 & 879 & $2.5 \mathrm{NaOH}$ & \\
\hline
\end{tabular}

In sehr anschaulicher Weise geht die Wirkung der Alkaliresp. Säure-Injection aus einer graphischen Darstellung der Athemgrössen hervor; es sei daher eine solche von dem letzten Versuche hier beigegeben.

Aus den Versuchsprotocollen geht unzweifelbaft hervor, dass die Alkali-Injectionen die Athemgrösse herabsetzten. Trotz der 
Ueb. den Einfluss von Alkali u. Säure auf die Erregung des A themcentrums. 297

Versuch VII vom 25. 6. 83.

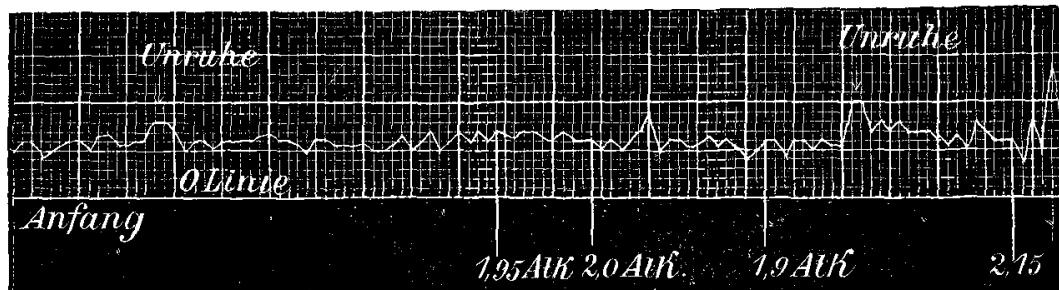

$1 \mathrm{~mm}^{\mathrm{m}} \mathrm{O}_{\text {Abscise }}=10 \mathrm{sec}$ Athmung
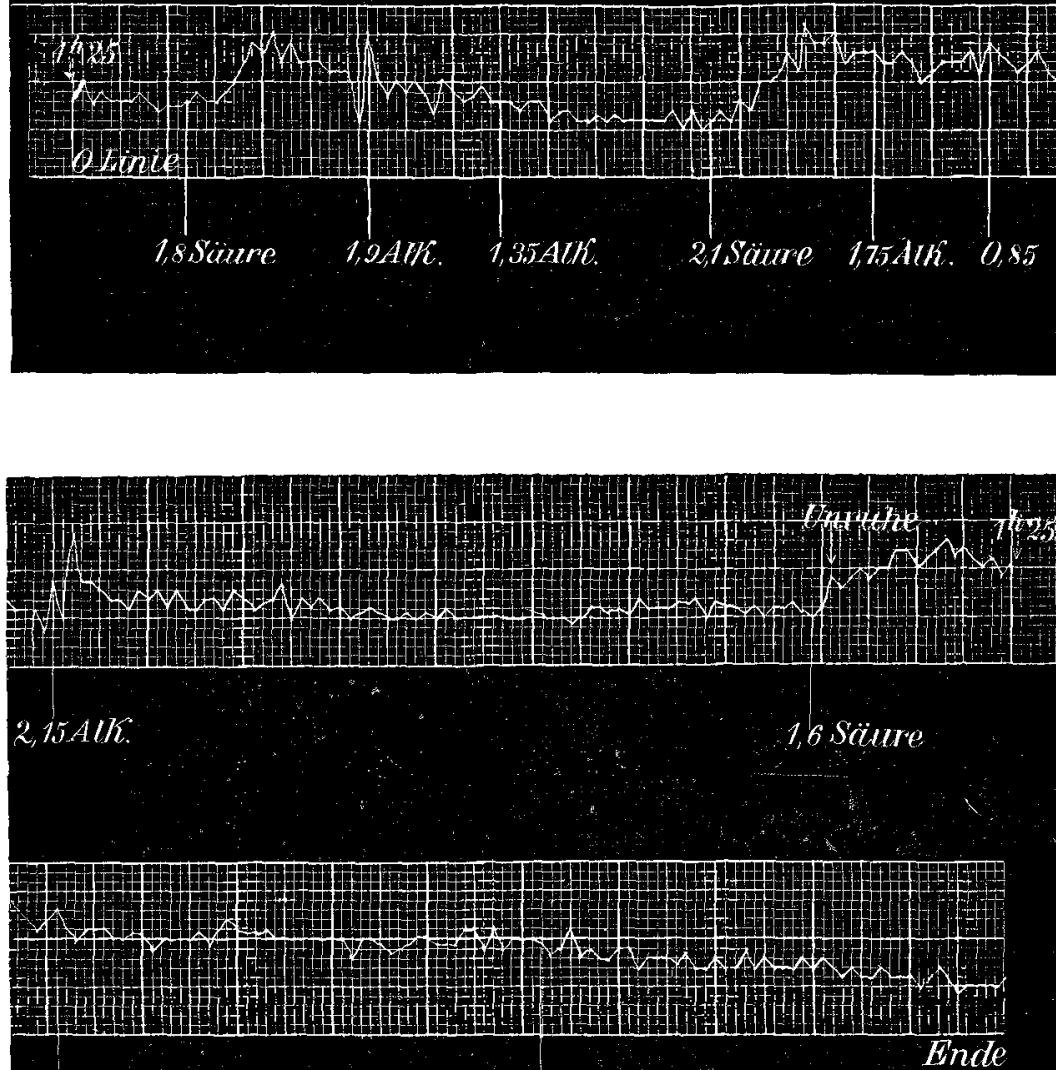
$0.85 \mathrm{AlK}$
$0.5 A l k$ 
oben erwähnten Gründe, welche zeigten, dass bei jeder Injection wie bei jedem Manipuliren am Thiere mehr oder weniger mittelbar auch ein erregendes Moment für die Athmung vorhanden sein muss, gelang es doch zweimal die Ventilationsgrösse durch Alkali unter den Werth herabzudrïcken, den sie an intacten Thiere hatte (vergl. Versuch II, Beobachtung 3 und 4). Die im ersten Moment nach einer Injection von Alkali erfolgenden beschleunigten und vertieften Athemzlge sind besonders im Hinblick auf die nur leichte Narcose der Thiere wohl erklärlich.

Im Gegensatz hierzu sahen wir die Athemcurven rapid und andauernd steigen, sowie Säure injicirt wurde. Dem Eindruck nach leistete der Athemapparat der Thiere das Maximum, zu dem er uberhaupt befähigt war; die Respirationen waren beschleunigt und vertieft. Erfolgte hierauf wieder Alkali-Injection, so konnte, wie besonders Versuch VII beweist, die Athemgrösse wieder zur Norm zurückgebracht werden.

Allerdings fällt auf, dass letzteres nicht durch eine der inji. cirten Säuremenge äquivalente Alkalimenge möglich war; von letzterer konnten die Thiere nicht nur iberhaupt mehr vertragen, sondern es verlangte auch die Aufhebung der Säurewirkung mehr Alkali, als zur Neutralisation der Säure nothwendig gewesen wäre. Andererseits seben wir, dass nach sebr erheblichen Alkaliinjectionen (Versuch VII, Beobachtung 2-4) relativ viel geringere Säureinjectionen sofort erregend und zwar maximal erregend wirken. Es bleibt hiernach wohl nur die Annahme übrig, dass die Ganglienzellen eben leichter eine schnelle Verstärkung der Alkalescenz des Blutes vertragen als eine schnelle Verminderung. Man wird wohl umsomehr zu dieser Annahme gedrängt, als das injicirte Alkali seiner relativ geringern Diffusionsfähigkeit wegen gewiss durch den Capillarkreislauf des einen Hinterschenkels eher weniger zurückgehalten wurde als die Säure, abgerechnet davon, dass die alkalischen Gewebe einen Theil der letzteren sicher bald neutralisirten.

Ueber den Grad der Reactionsänderung des Blutes durch die in den vorstehend mitgetheilten Versuchen ausgeführten Injectionen ist kaum etwas Genaueres festznstellen. Bei den ersten drei Versuchen, in welchen die Injection in eine Carotis erfolgte und dann von dem Aortenbogen aus die Fliissigkeit direct dem Hirn zugeleitet wurde, ist absolut nichts auszusagen, abgesehen von der 
Ueb. den Einfluss von Alkali u. Säure auf die Erregung des Athemcentrums. 299

oben ausgesprochenen Vermuthung, dass Injectionsflissigkeit und Blut sich nicht stets vollkommen gemischt haben mögen.

In den letzten 4 Versuchen könnte man daran denken; die infundirten Alkali- resp. Säure-Aequivalente mit der Alkalescenz der gesammten Blutmasse der Thiere zu vergleichen. Mit einer solchen Rechnung ist aber nur eine ungefähre Orientirung mit breiten Fehlergrenzen zu erreichen. Einmal ist nicht festzustellen, in wie weit die Gewebe (des einen Hinterschenkels der Gefässwandungen und der Lunge) von dem Alkali oder der Säure absorbirten, endlich zeigen zahlreiche Versuche, dass normal bei den Thieren der Grad der Alkalescenz des Blutes grossen Schwankungen unterworfen ist. Zuntz fand bei Hunden in $100 \mathrm{gr}^{1}$ ) Blut durch Titriren die Alkalescenz schwankend zwischend 2,51-5,17 Milligramm-Aequivalenten Alkali, d. h. um über das Doppelte. Lassar ${ }^{2}$ ) fand, ebenfalls durch Titriren, die Alkalescenz von $100 \mathrm{gr}$ Blut normaler deutscher Kaninchen, welche sogar längere Zeit vorher unter gleichen Bedingungen (gleichem Futter etc.) gehalten worden waren, zwischen 3,35 und 5,54 Milligramm-Aequivalenten schwankend.

In Anbetracht dieser Unsicherbeiten kann also, wie gesagt, die folgende Berechnung aus den letzten drei Versuchen nur eine ganz ungefähre Schätzung der Reactionsschwankungen des Blntes der Versuchsthiere liefern.

Es wurde dabei angenommen, dass die Thiere $1 / 13$ ibres Lebendgewichtes an Blut besessen haben und letzteres in seiner Alkalescenz dem mittleren von $L$ as s a r (l. c.) gefundenen Werthe entsprach, nämlich in $100 \mathrm{gr}=4,72$ Milligramm-Aequivalente Alkali.

Das Thier in Versuch 5 wog $1050 \mathrm{gr}$, dürfte daher $81 \mathrm{gr}$ Blut enthalten haben, dessen Alkalescenz etwa 3,82 Milligramm-Aequivalenten entsprach. Die drei ersten Injectionen von Natron, welche im Laufe von $3 \frac{2}{3}$ Minuten gemacht wurden (Beobachtungsdauer in Sumpa $=405$ Sekunden $=6,7$ Minuten), erniedrigten die Athemgrösse zuletzt fast um $30 \%$. Es waren 4,4 Milligramm-Aequivalente injicirt worden, somit eine grössere Alkalimenge, als der gesammten Blutalkalescenz entsprach. Die darauf folgende Säure-

1) Zuntz: „Die Gase des Blutes etc. - Hermann, Handbuch der Physiol. Bd. 3, p. 73.

2) Lassar, Pfüger's Archiv, Bd. IX, p. 49 u. s. w. 
injection von 2,1 Milligramm-Aequivalenten müsste immer noch einen Alkaliüberschuss von 2,3 Alkali, d. h. von $66 \%$ der normalen Blutalkalescenz im Thiere zurücklassen, trot\%dem wurde sofort die Atbmung gegen die anfänglich beobachtete normale Grösse un über $30 \%$, gegenüber der zuletzt beobachteten Grösse um über $100 \%$ gesteigert.

Zur Abschwächung des Säurereizes auf die Athemcentren genügte hierauf nicht eine Neutralisation der Säure, sondern kaum cin Ueberschuss von 1,9 Milligramm-Aequivalenten Alkali, d. h. beinahe die doppelte Menge.

Die hierauf erfolgende starke Säureinjection, obgleich sie lange nicht genügte, die vorher infundirten Alkalimengen zu neutralisiren, tödtete nach einer kurzen, abcrmaligen starken Beschleunigung der Athmung das Thier.

In Versuch 6 wog das Thier $1510 \mathrm{gr}$, enthielt also etwa $116 \mathrm{gr}$ Blut mit einer Alkalescenz von 5,48 Milligramm-Aequivalenten. Letætere durch Säure auf 4,93 , d. b. um $10 \%$ erniedrigt, ergaben beinahe Verdoppelung der Athemgrösse, weitere Erniedrigung auf 4,08 , d. h. um $26,4 \%$ steigerten die Athemgrösse um mehr als $100 \%$ und erst nach 4 Minuten klang die Wirkung des Säurereizes erheblich ab.

Im letzten Versuch 7 sind ganz ähnliche Resultate zu bemerken. Das Thier, $1360 \mathrm{gr}$ schwer, enthielt $105 \mathrm{gr}$ Blut mit etwa 4,16 Milligramm-Aequivalenten Alkali.

Die Injectionen brachten in Milligramm-Aequivalenten in das Thier :

\begin{tabular}{|c|c|c|c|c|c|}
\hline \multirow{2}{*}{$\begin{array}{c}\text { Zahl } \\
\text { der Injectionen }\end{array}$} & \multicolumn{2}{|r|}{ Alkali- } & \multirow{2}{*}{$\begin{array}{l}\text { Säure- } \\
\text { quivalente }\end{array}$} & \multicolumn{2}{|c|}{ In Procent } \\
\hline & & Milligr.-A & & Alkali & Säure \\
\hline 4 & $=$ & 8,0 & - & $161,3 \%$ & - \\
\hline 2 & $=$ & - & 3,4 & - & $68 \%$ \\
\hline 2 & $=$ & $3,2.5$ & - & $65 \%$ & $40,5 \%$ \\
\hline 1 & $=$ & 一 & 2,1 & $110 \%$ & - \\
\hline 3 & $=$ & 5,5 & - & - & - \\
\hline & & 16,75 & 5,5 & & \\
\hline
\end{tabular}

Durch diese Injection war die Alkalescenz des Blutes (wenn die kaum zulïssige Annahme gemacht wird, dass die Gewebe des Thieres von der Reaction auszuschliessen wären), nach Abzug der durch die Säuren neutralisirten Mengen von 4,96 auf 16,21 Milli- 
Ueb. den Einfluss von Alkali u. Säure auf die Erregung des Athemcentrums. 301

gramm-Aequivalente gebracht, also mehr als verdreifacht worden und obgleich aus der oben mitgetheilten Tabelle hervorgeht, dass stets die Alkaliinjectionen beruhigend wirken, die durch die Säure gesetzte Erregung herabstimmen, ist doch am Schlusse des Experimentes nur knapp die Athemgrösse wieder erreicht worden, welche das Thier anfänglich normal zeigte. Der Grad der Steigerung der Athemgrösse durch Säure war auch in diesem Versuche etwa $100 \%$.

Vergleicht man die in den geschilderten Versuchen durch Säureinjectionen hervorgebrachte Verminderung der Alkalescenz des Blutes, wobei also maximale Respiration erfolgte, mit den letzthin von Zuntz and Geppert mitgetheilten Werthen (dies Arch. Bd.XLII S. 233) iuber die Verminderung der Alkalecenz des Blutes durch starke Muskelactionen, so erhält man nicht sehr von einander abweichende Zahlen.

Genannte dutoren fanden in drei Titrirversuchen die Alkalescenz des Blutes nach Jagen resp. Tetanisiren des Thieres um 15 bis $56 \%$, im Mittel um $43 \%$ gegenüber der Blutalkalescenz des ruhenden Thieres herabgesetzt; ich verminderte durch die Injection z. B. in Versuch 6 die Alkalescenz um $10 \%$ resp. $27 \%$, in Versuch 7 um $68 \%$ resp. $40 \%$; in Versuch 5 wurde durch stärkere Säureinjectionen das Thier schliesslich getödtet.

Beim Kaninchen wenigstens ist biernach unbestreitbar trotz aller Vorbebalte in Bezug auf dic Fellergrenzen dervorstehenden Berechnu g zu behaupten, dass diedureb die Muskelthätig keit erfolgende Acidulirung des Blutes einen sehrerheblichen Antheilander Erregung des Athemcentrums haben uns

Neben diesem Hauptresultat vorstehender Untersuchungen seien noch folgende Schlüsse hervorgehoben.

1. Die Ganglienzellen, die die Innervation des Athemapparates bewirken (wahrscheinlich auch die ïbrigen), werden durch eine Verstärkung der Blutalkalescenz in geringerem Grade beeinflusst, als durch eine äquivalente Verminderung derselben.

2. Die durch Verminderung der Blutalkalescenz hervorgebrachte Reizung der Athemcentren kann durch Alkaliinjectionen aufgehoben werden.

3. Es hat den Anschein, dass die Erregung des AthemcenE. Plïger, Archiv f. Physiologie. Ba. xLIt. 
trums mehr noch durch die Schwankung der Blutreaction in der Richtung verminderter Alkalescenz hervorgebracht wird, als durch den absoluten Gehalt des Blutes an alkalischen Affinitäten, so dass innerhalb gewisser Grenzen sich die Reizbarkeit der venösen Centralapparate wechselnder Alcalescenz des Blutes anzupassen vermag. Wie weit diese Grenzen zu ziehen sind, ist aus den obigen Versuchen um deswillen nicht zu erschliessen, als keine Daten gewonnen worden sind, die einen Anhalt über die Zerstörung der injicirten organischen Säure durch die Gewebe, resp. uber eine Neutralisation derselben durch Gewebsbestandtheile geben. Die verhältnissmässig nicht sehr lange Zeit, in welcher sich nach einer Säureinjection die Athemgrösse wieder der normalen näherte, ohne dass später Alkali eingeführt worden war (vergl. Vers. 6), kann sehr plausibel durch die Annahme erklärt werden, dass der $\mathrm{Or}^{-}$ ganismus bald einen belangreichen Theil der Säure entfernt hat; keinesfalls ist man zu dem Schlusse genöthigt, dass sich die nervösen Apparate so rasch an eine erheblich veränderte Blutalkalescenz gewöhnt hätten.

Es liegt nahe, die längst bekannte Reizwirkung der Kohlensäure als einen speciellen Fall der von mir constatirten Wirkung. von Säuren im Allgemeinen zu betrachten.

Für die freundliche Unterstïtzung bei den Versuchen sage ich Herm Professor Dr. Z u n $\mathrm{z}$ und Herrn Dr. B. Tacke meinen besten Dank. 